\title{
Clinical-radiological correlations in COVID-19-related venous thromboembolism: Preliminary results from a multidisciplinary study
}

\author{
Maddalena Alessandra $\mathrm{Wu}^{1}$ (D) | Riccardo Colombo ${ }^{2}$ (D) | Massimo Arquati $^{3}$ | \\ Sonia Ippolito $^{4}$ | Alba Taino $^{1}$ | Diego Ruggiero ${ }^{5}$ | Francesca Tonelli ${ }^{1}$ | \\ Lucia Trombetta $^{1}$ | Pietro Facchinetti ${ }^{1}$ | Pierluigi Glielmo ${ }^{6}$ | Chiara Cogliati ${ }^{1}$ | \\ Nicola Flor ${ }^{4}$
}

\author{
${ }^{1}$ Division of Internal Medicine, ASST \\ Fatebenefratelli Sacco, Luigi Sacco Hospital, \\ University of Milan, Milan, Italy \\ ${ }^{2}$ Division of Anesthesiology and Intensive \\ Care, ASST Fatebenefratelli Sacco, Luigi \\ Sacco Hospital, University of Milan, Milan, \\ Italy \\ ${ }^{3}$ Division of Emergency Medicine, ASST \\ Fatebenefratelli Sacco, Luigi Sacco Hospital, \\ University of Milan, Milan, Italy \\ ${ }^{4}$ Department of Radiology, ASST \\ Fatebenefratelli Sacco, Luigi Sacco Hospital, \\ University of Milan, Milan, Italy \\ ${ }^{5}$ Division of Cardiology, ASST \\ Fatebenefratelli Sacco, Luigi Sacco Hospital, \\ University of Milan, Milan, Italy \\ ${ }^{6}$ Postgraduation School in Radiodiagnostics, \\ Facoltà di Medicina e Chirurgia, University \\ of Milan, Milan, Italy

\section{Correspondence} \\ Riccardo Colombo, Division of \\ Anesthesiology and Intensive Care, ASST \\ Fatebenefratelli Sacco, Luigi Sacco Hospital, \\ University of Milan, Via GB Grassi 74, 20157, \\ Milan, Italy. \\ Email: riccardo.colombo@asst-fbf-sacco.it; \\ riccardo.colombo@unimi.it
}

\begin{abstract}
Introduction: Among the multiple complex pathophysiological mechanisms underlying COVID-19 pneumonia, immunothrombosis has been shown to play a key role. One of the most dangerous consequences of the prothrombotic imbalance is the increased incidence of micro- and macrothrombotic phenomena, especially deep vein thrombosis (DVT) and pulmonary embolism (PE).

Methods: We investigated the correlation between radiological and clinicalbiochemical characteristics in a cohort of hospitalised COVID-19 patients.

Results: PE was confirmed in 14/61 (23\%) patients, five (35.7\%) had DVT. The radiographic findings, quantified by Qanadli score calculated on CT angiography, correlated with the clinical score and biochemical markers. The ratio between the right and left ventricle diameter measured at CT angiography correlated with the length of hospital stay.

Conclusion: In our cohort radiological parameters showed a significant correlation with clinical prognostic indices and scores, thus suggesting that a multidisciplinary approach is advisable in the evaluation of PE in COVID-19 patients.
\end{abstract}

\section{1 | INTRODUCTION}

Recent studies demonstrate that severe COVID-19 patients are at high risk for venous thromboembolism (VTE) and mortality. ${ }^{1}$ The outbreak of the pandemic led physicians to work in multidisciplinary specialised teams worldwide so that the need for an integrated approach matching clinical, laboratory and radiological data became undeniable.
In the setting of an acute potentially rapidly evolving infectious disease, with concerns related not only to individual patient's outcome but also to risks for healthcare operators, the correct risk stratification and prognostic evaluation is of paramount importance.

Our study aimed at analysing the relationship between radiological findings and clinical characteristics in hospitalised COVID-19 patients who developed pulmonary embolism (PE). 


\section{2 | MATERIALS AND METHODS}

Observational retrospective cohort study carried out at Luigi Sacco Hospital in Milan, the referral centre for highly transmissible diseases in Northern Italy. All patients admitted to COVID-19 specialised wards (Internal Medicine and ICU) between 28 March and 6 June 2020 were considered. The study adhered to the principles of the Declaration of Helsinki for medical research involving human subjects was approved by local IRB, and written informed consent was acquired from all subjects or their surrogates.

Within the patients' list, we identified subjects hospitalised because of respiratory symptoms, with SARS-CoV-2 infection confirmed by real-time PCR on naso-pharyngeal swabs.

Demographic-clinical characteristics and laboratory data at admission were extracted from the digital medical charts of our hospital.

As per usual routine, locally defined clinical practice, to assess signs of deep vein thrombosis (DVT), all patients underwent lower limbs venous compression ultrasound (CUS) at admission.

All CT chest angiograms performed were extracted from the hospital picture archiving and communication system database.

All the CT pulmonary angiograms were acquired on a 64 row multidetector CT (GE HealthCare, Chicago, IL) after injection of $80 \mathrm{~mL}$ of high concentration iodine contrast agent at a flow rate of $4 \mathrm{~mL} / \mathrm{s}$ with the use of a bolus-tracking technique. Images were reconstructed with a slice thickness of $1 \mathrm{~mm}$ in a mediastinal and parenchymal window. Two chest radiologists (with 20 and 15 years of experience), blinded to patient status as well as clinical and biological features, independently analysed all CT examinations searching for PE.

Moreover, they calculated the Qanadli score. To define the CT obstruction index (Qanadli score), the arterial tree of each lung was regarded as having 10 segmental arteries (three to the upper lobes, two to the middle lobe and the lingula and five to the lower lobes). The presence of embolus in a segmental artery was scored 1 point, and emboli in the most proximal arterial level were scored a value equal to the number of segmental arteries arising distally. To provide additional information about the residual perfusion distal to the embolus, a weighting factor was assigned to each value, depending on the degree of vascular obstruction. This factor was equal to 0 , when no thrombus was observed; 1 , when partially occlusive thrombus was observed or 2 , with total occlusion. Thus, the maximal CT obstruction index was 40 per patient. Isolated subsegmental embolus was considered as partially occluded segmental artery and was assigned a value of 1 . The percentage of vascular obstruction was calculated by dividing the patient score by the maximal total score and by multiplying the result by 100 . Therefore, the CT obstruction index can be expressed as follows: $(n \times d) / 40 \times 100$, where $n$ is the value of the proximal thrombus in the pulmonary arterial tree equal to the number of segmental branches arising distally (minimum, 1; maximum, 20), and $d$ is the degree of obstruction (minimum, 0; maximum, 2). In case of discordance, a simultaneous reading to reach consensus was achieved.

The Qanadli score provides an accurate but simple tool to quantify the presence, location and degree of arterial obstruction on CT images, able to highlight the burden of PE, thus distinguishing massive from submassive PE. ${ }^{2,3}$

The revised Geneva score (rGeneva) was calculated for all patients with DVT and PE and the Pulmonary Embolism Severity Index (PESI) score for all cases of PE.

The rGeneva clinical prediction rule is useful to estimate the pretest probability of PE and stratify patients into low, intermediate or high risk based on medical history and physical exam alone. ${ }^{4,5}$ It aids in reducing unnecessary imaging studies by identifying low-risk patients who can be ruled out for PE with a negative D-dimer serum test.

The PESI score is a risk stratification tool that has been validated to assess the probability of overall mortality and early outcome of patients with newly diagnosed PE. ${ }^{4,6-8}$ This can help physicians to make decisions on the appropriate management distinguishing patients who could potentially be treated as outpatients, from those who could benefit from higher levels of care.

All patients received low molecular weight heparin at prophylactic dosage.

Data are presented as mean (range), or number and percentage, as appropriate. Correlations were assessed with non-parametric Spearman's $r$, and $P<.05$ was considered to be statistically significant. Categorical variables were assessed with Fisher's exact test. Statistical analysis was carried out using GraphPad Prism 8 (GraphPad Software, San Diego, CA) and SPSS 26 (IBM Corp., Armonk, NY).

\section{3 | RESULTS}

In the study period, 145 patients underwent chest CT angiographies because of respiratory symptoms, and 61 of them (42\%) were positive for SARS-CoV-2.

CT angiography revealed PE in 14 COVID-19 patients (23\%). No differences were highlighted in interstitial pulmonary involvement between COVID-19 patients with and without PE. An example of CT finding is shown in Figure 1.

Data extraction from our hospital database revealed that last year we found $18 \mathrm{PE}$ cases over 112 CT angiograms (16\%) performed during the same time span.

Table 1 shows the characteristics of patients with confirmed PE. None had a history of previous DVT or PE, one patient had a recent history of bone fracture, and two patients had undergone recent surgical intervention. Eight patients (57.1\%) had been hospitalised within the previous 3 months.

CUS revealed DVT in five PE cases (35.7\%).

Three patients out of 14 patients (21\%) with COVID-19 pneumonia and pulmonary embolism detected by CT angiography had pulmonary infarction.

In one patient PE was massive and induced severe haemodynamic compromise, requiring thrombolysis and timely admission to ICU.

Qanadli score had a significant correlation with PESI, D-dimer, serum high-sensitivity troponin, serum albumin, arterial pressure of 


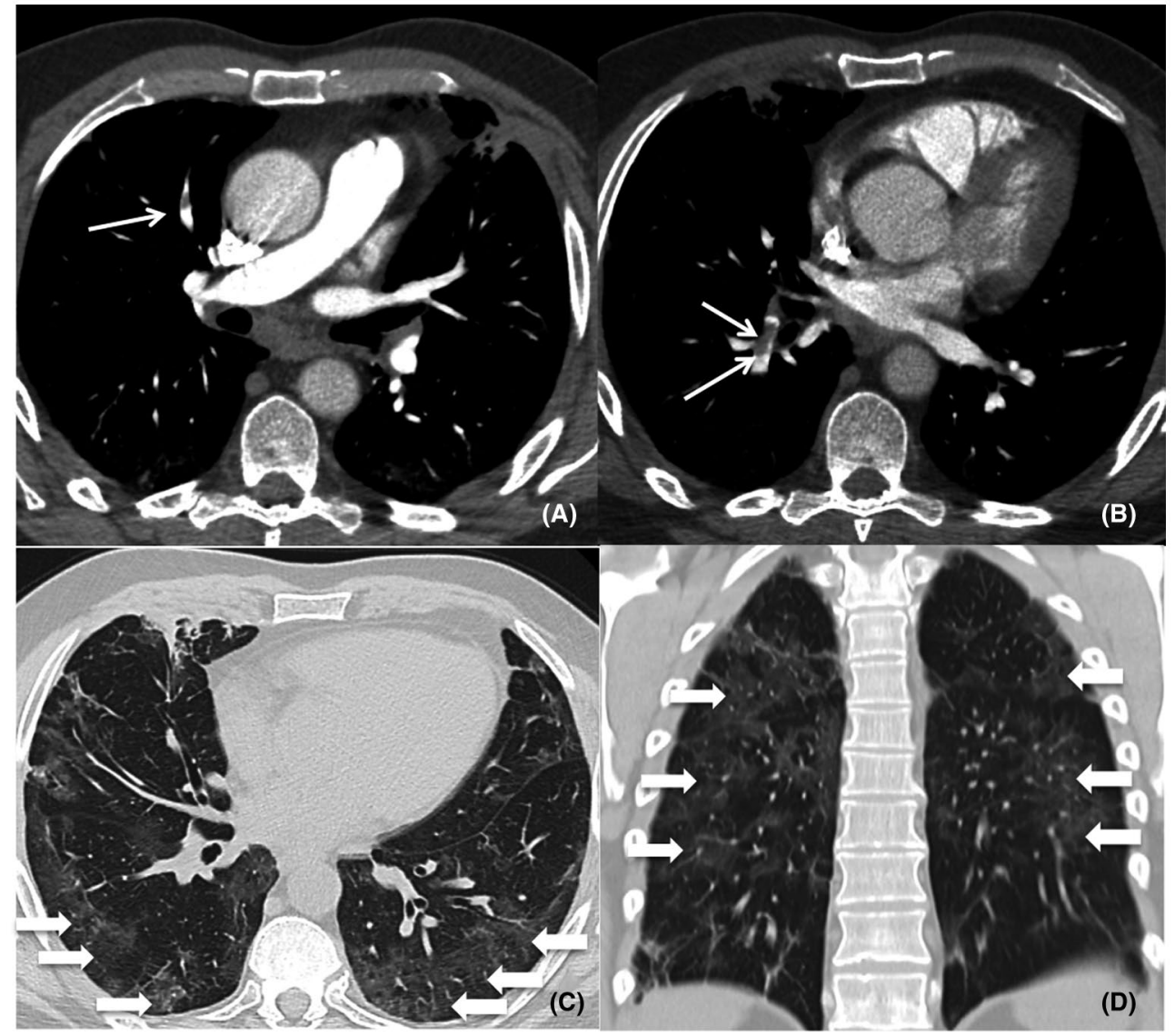

FIGURE 1 Pulmonary CT angiography of a 59-year-old male. The CT scan was obtained 37 days after the onset of COVID-19 symptoms and on the day the patient was admitted to ED. Axial CT images ( $A-B$, mediastinum windows) show right segmental pulmonary embolism (thin arrows). Axial and coronal CT images (C-D, lung windows) show bilateral ground-glass opacities (arrows) with typical posterior peripheral distribution

oxygen to inspired fraction of oxygen ratio $\left(\mathrm{pO}_{2} / \mathrm{FiO}_{2}\right)$ and length of hospital stay (Figure 2). PESI had a significant correlation with albu$\min (r=-0.655,95 \% \mathrm{Cl},-0.884$ to $-0.174, P=.013)$ and length of hospital stay ( $r=0.728,95 \% \mathrm{Cl} 0.307$ to $0.911, P=.004)$.

Furthermore, the ratio between the right and left ventricle diameter $\left(V_{R} / V_{L}\right)$ measured at CT scan ${ }^{9}$ correlated with the length of hospital stay ( $r=0.719,95 \% \mathrm{Cl}, 0.189$ to $0.924, P=.015)$.

All studied patients survived.

\section{4 | DISCUSSION}

Our study found that the Qanadli score, a radiological index of PE burden, correlated with the severity of clinical findings and biochemical-metabolic alterations in COVID-19 patients. In fact, in our cohort, this radiological score showed good correlations with the degree of respiratory dysfunction and the troponin value as a marker of myocardial injury.

Moreover, in accordance with previous studies, ${ }^{10}$ Qanadli score as well as the CT estimation of the right ventricle overload, expressed as $V_{R} / V_{L}$ ratio, correlated with the clinical impact of $P E$, as shown by the length of hospital stay.
In our cohort, patients who developed VTE (either DVT or PE) had a pretest probability in the intermediate-high range, as also confirmed by the rGeneva score. The spectrum of mortality risk assessed by PESI score was wide, ranging from class I to class $V$ risk strata, but no patients died in our cohort.

The strong correlation between radiological scores, such as Qanadli and $V_{R} / V_{L}$ ratio, and the clinical impact of $P E$ underlines the value of the radiological contribution in a multidisciplinary approach to provide COVID-19 patients with the most appropriate and timely management. In fact, our results support the strong bidirectional link between clinical-biochemical and radiological data and the need for an integrated view: the collection of a thorough medical history and the estimation of the pre-test probability of each patient should be the starting point, to which laboratory data add further insights. These steps should guide the appropriate decision on whether CT angiography is required; when CT angiography is performed and PE is identified, accurate evaluation of images (with the help of a score) can allow to obtain thorough indications on the overall burden of PE. From this multidisciplinary approach stems the possibility to draw a whole picture of each patient, which can help to tailor management not only in the acute phase but also in the long term. 
TABLE 1 Characteristics of the studied subjects

\begin{tabular}{|c|c|c|c|c|}
\hline Variable & Whole population $(n=14)$ & Positive CUS ( $n=5$ ) & Others $(n=9)$ & $P$ \\
\hline WBC $\left(10^{3}\right.$ cells $\left./ \mathrm{mL}\right)$ & $10.89(6.96-23.69)$ & $11.56(6.96-23.69)$ & $10.52(6.99-14.62)$ & .518 \\
\hline Lymphocytes $\left(10^{3}\right.$ cells $\left.\cdot \mathrm{mL}\right)$ & $1.34(0.360-3.45)$ & $0.76(0.36-1.12)$ & $1.67(0.58-3.45)$ & .06 \\
\hline Hemoglobin (g/dL) & $19.3(7.8-114)$ & $11.3(7.8-13.7)$ & $23.7(10.3-114)$ & .298 \\
\hline D-dimer (ng/mL) & 7092 (448-26 362) & $13646(4732-26362)$ & $3450(448-6447)$ & .019 \\
\hline PT (INR) & $1.22(1.03-1.48)$ & $1.32(1.19-1.45)$ & $1.17(1.03-1.48)$ & .06 \\
\hline PTT (Ratio) & $1.18(0.93-1.38)$ & $1.2(1.02-1.35)$ & $1.16(0.93-1.38)$ & .797 \\
\hline Fibrinogen (mg/dL) & $5.53(3.04-7)$ & $4.92(3.04-5.97)$ & $5.8(4.06-7)$ & .26 \\
\hline CRP (mg/L) & $47(1-246)$ & $42(1-101)$ & $50(1-247)$ & .797 \\
\hline Arterial pH & $7.47(7.42-7.52)$ & $7.46(7.44-7.49)$ & $7.47(7.42-7.52)$ & .699 \\
\hline Arterial $\mathrm{pCO}_{2}(\mathrm{~mm} \mathrm{Hg})$ & $35.3(25-47)$ & $31.4(25-36)$ & $37.4(29-47)$ & .147 \\
\hline Arterial $\mathrm{pO}_{2}(\mathrm{~mm} \mathrm{Hg})$ & $72(38-121)$ & $62(38-81)$ & $79(54-121)$ & .19 \\
\hline Arterial lactate (mmol/L) & $1.3(0.6-3.3)$ & $1.5(0.7-3.3)$ & $1.2(0.6-1.7)$ & 1 \\
\hline Arterial Sat (\%) & $95.6(88-100)$ & $94.7(89-99)$ & $96(88-100)$ & .71 \\
\hline $\mathrm{FiO}_{2}$ & $0.387(0.21-0.8)$ & $0.45(0.21-0.8)$ & $0.34(0.21-0.8)$ & .606 \\
\hline PEEP $\left(\mathrm{cmH}_{2} \mathrm{O}\right)$ & $0(0-0)$ & $0(0-0)$ & $0(0-0)$ & 1 \\
\hline $\mathrm{PaO}_{2} / \mathrm{FiO}_{2}$ & $271.8(47-576)$ & $209.2(47-361)$ & $306.6(97-576)$ & .364 \\
\hline GENEVA & $5.5(3-9)$ & $6.8(5-9)$ & $4.8(3-9)$ & .06 \\
\hline Qanadli & $17.9(0-75)$ & $30(7.5-75)$ & $12.5(0-27.5)$ & .33 \\
\hline$V_{R} / V_{L}$ & $0.93(0.7-1.6)$ & $0.95(0.7-1.6)$ & $0.91(0.8-1.07)$ & .315 \\
\hline Length of hospital stay (days) & $22.2(6-64)$ & $30.8(7-64)$ & $17.4(6-37)$ & .518 \\
\hline Albumin (g/dL) & $2.8(2.1-3.4)$ & $2.5(2.1-2.7)$ & $3(2.2-3.4)$ & .19 \\
\hline $\begin{array}{l}\text { Time from symptoms onset to CT scan } \\
\text { study (days) }\end{array}$ & $22.1(1-43)$ & $22.4(5-43)$ & $21.9(1-43)$ & .898 \\
\hline Time from admission to CT scan (days) & $2.1(0-16)$ & $2.8(0-14)$ & $1.8(0-16)$ & .898 \\
\hline
\end{tabular}

Note: Values are shown as mean (range).

We speculate that the correlation found between Qanadli score and albumin values is not negligible. We have recently provided insights into the role of increased endothelial permeability in the pathogenesis of COVID-19, of which hypoalbuminemia is one of the useful markers. ${ }^{11}$ Therefore, the results presented highlight the complex mechanisms underlying the so-called immunothrombosis which develops in COVID-19, including endothelial damage, abnormal blood flow dynamics, hypercoagulability state, platelet-neutrophil interactions and release of Neutrophil Extracellular Traps or even autoimmune mechanisms. ${ }^{12}$
A limitation of our study is that many critically ill patients did not undergo CT angiography because of the marked disproportion between available resources and demand as well as risks related to transport for both patients themselves and healthcare professionals taking care of them during the surge of the epidemic. We plan to expand our cohort to provide results on the correlation between the Qanadli score and major outcomes, such as mortality and morbidity, as well as the rate of ICU admission.

Our results strongly suggest that a multidisciplinary approach integrating radiological and clinical-laboratory findings is of pivotal 

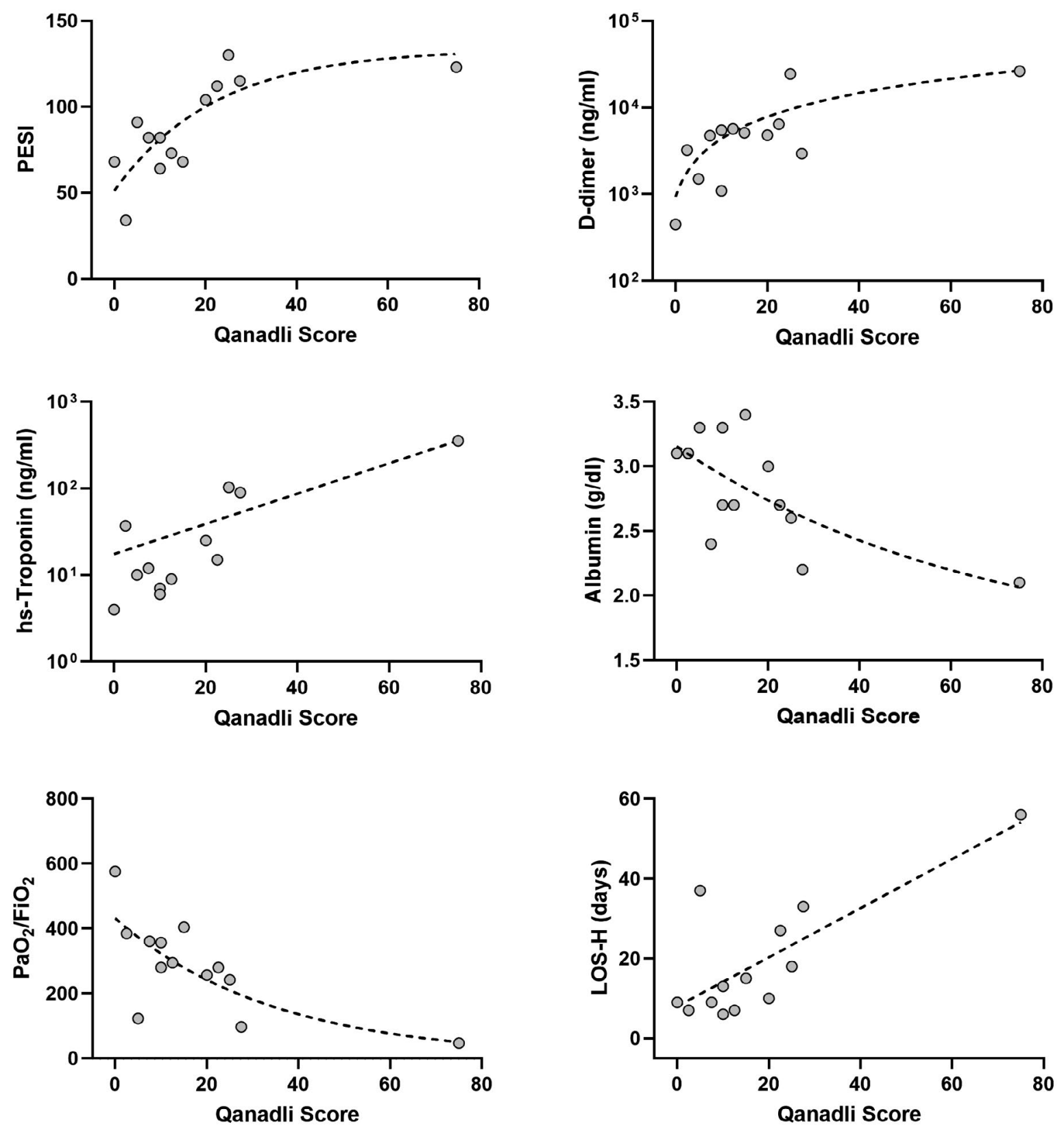

FIGURE 2 Correlation between radiological score of thromboembolic burden (Qanadli score) and clinicalbiochemical markers. Correlation of Qanadli score with PESI ( $r=0.774,95 \% \mathrm{Cl} 0.372$ to $0.931, P=0.003)$, D-dimer $(r=0.679,95 \% \mathrm{Cl} 0.188$ to $0.899, P=.013)$, high-sensitivity troponin $(r=0.683,95 \% \mathrm{Cl} 0.161$ to $0.906, P=0.017)$, serum albumin $(r=-0.592,95 \% \mathrm{Cl}-0.867$ to $-0.043, P=0.036)$, arterial pressure of oxygen to inspired fraction of oxygen ratio $\left(\mathrm{pO}_{2} / \mathrm{FiO}_{2}\right)(r=-0.687,96 \% \mathrm{Cl}-0.902$ to $-0.202, P=0.011)$, and length of hospital stay $(r=0.585,95 \% \mathrm{Cl} 0.032$ to $0.864, P=.038)$. hs-Troponin, high sensitivity troponin; LOS-H, length of hospital stay

importance in the evaluation and management of PE in COVID-19 patients.

\section{DISCLOSURES}

The authors have no conflicts of interest to declare.

\section{AUTHOR CONTRIBUTIONS}

Maddalena Alessandra Wu: Conceptualisation (lead); Data curation (lead); Investigation (equal); Methodology (equal); Writing-original draft (lead). Riccardo Colombo: Data curation (lead); Formal analysis (lead); Investigation (equal); Methodology (equal); Validation (lead); Writing-original draft (equal). Massimo Arquati: Data curation (equal); Writing-review and editing (equal). Sonia Ippolito: Investigation (equal); Writing-review and editing (equal). Alba Taino: Data curation (equal); Writing-review and editing (equal). Diego Ruggiero: Data curation (equal); Writing-review and editing (equal). Francesca Tonelli: Data curation (equal); Writingreview and editing (equal). Lucia Trombetta: Data curation (equal); Writing-review and editing (equal). Pietro Facchinetti: Data curation (equal); Writing-review and editing (equal). Pierluigi Glielmo: Data curation (equal); Writing-review and editing (equal). Chiara Cogliati: Data curation (equal); Formal analysis (equal); Investigation 
(equal); Supervision (equal); Validation (equal); Writing-review and editing (equal). Nicola Flor: Conceptualisation (lead); Data curation (lead); Investigation (equal); Methodology (equal); Supervision (lead); Validation (lead); Writing-original draft (equal).

\section{DATA AVAILABILITY STATEMENT}

Data are available on reasonable request and after Institutional Ethical Committee authorisation.

\section{ORCID}

Maddalena Alessandra Wu iD https://orcid.

org/0000-0003-1078-1632

Riccardo Colombo (iD https://orcid.org/0000-0002-9616-803X

\section{REFERENCES}

1. Connors JM, Levy JH. COVID-19 and its implications for thrombosis and anticoagulation. Blood. 2020;135:2033-2040.

2. Ghaye B, Ghuysen A, Bruyere P-J, et al. Can CT pulmonary angiography allow assessment of severity and prognosis in patients presenting with pulmonary embolism? What the radiologist needs to know. Radiographics. 2006;26:23-39; discussion 39-40.

3. Qanadli SD, El Hajjam M, Vieillard-Baron A, et al. New CT index to quantify arterial obstruction in pulmonary embolism: comparison with angiographic index and echocardiography. AJR Am J Roentgenol. 2001;176:1415-1420.

4. Konstantinides SV, Meyer G, Becattini C, et al. 2019 ESC Guidelines for the diagnosis and management of acute pulmonary embolism developed in collaboration with the European Respiratory Society (ERS). Eur Heart J. 2020;41:543-603.

5. Penaloza A, Verschuren F, Meyer G, et al. Comparison of the unstructured clinician gestalt, the wells score, and the revised Geneva score to estimate pretest probability for suspected pulmonary embolism. Ann Emerg Med. 2013;62:117-124 e2.

6. Chan CM, Woods C, Shorr AF. The validation and reproducibility of the pulmonary embolism severity index. J Thromb Haemost. 2010;8:1509-1514.

7. Elias A, Mallett S, Daoud-Elias M, et al. Prognostic models in acute pulmonary embolism: a systematic review and meta-analysis. BMJ Open. 2016;6:e010324.

8. Kohn CG, Mearns ES, Parker MW, et al. Prognostic accuracy of clinical prediction rules for early post-pulmonary embolism all-cause mortality: a bivariate meta-analysis. Chest. 2015;147:1043-1062.

9. Ammari Z, Hasnie AA, Ruzieh M, et al. Prognostic value of computed tomography versus echocardiography derived right to left ventricular diameter ratio in acute pulmonary embolism. Am J Med Sci. 2021;361:445-450. https://doi.org/10.1016/j.amjms.2020.07.008

10. Furlan A, Aghayev A, Chang C-C, et al. Short-term mortality in acute pulmonary embolism: clot burden and signs of right heart dysfunction at CT pulmonary angiography. Radiology. 2012;265:283-293.

11. Wu MA, Fossali T, Pandolfi L, et al. Hypoalbuminemia in COVID-19: assessing the hypothesis for underlying pulmonary capillary leakage. J Intern Med. 2021;289:861-872. https://doi.org/10.1111/ joim.13208

12. Middleton EA, He X-Y, Denorme F, et al. Neutrophil extracellular traps contribute to immunothrombosis in COVID-19 acute respiratory distress syndrome. Blood. 2020;136:1169-1179.

How to cite this article: Wu MA, Colombo R, Arquati M, et al. Clinical-radiological correlations in COVID-19-related venous thromboembolism: Preliminary results from a multidisciplinary study. Int J Clin Pract. 2021;00:e14370. https://doi.org/10.1111/ijcp.14370 